\title{
La importancia de los métodos de imagen en el diagnóstico del carcinoma hepatocelular. Rol actual de la PET-CT
}

\section{The importance of diagnostic imaging on hepatocellular carcinoma.}

\section{Actual role of PET-CT}

Christian A. Zamora-Soberón*, Daniela Angulo-Salazar, Mary C. Herrera-Zarza y José L. Criales-Cortés

\section{RESUMEN}

El carcinoma hepatocelular (CHC) es actualmente la sexta neoplasia más frecuente a nivel mundial y la tercera causa de muerte relacionada con el cáncer. La tasa de supervivencia depende entre otras cosas de la carga y tamaño tumoral, además de un tratamiento oportuno. Actualmente, el ultrasonido constituye la principal herramienta de screening en pacientes con hígado cirrótico, en los que el riesgo de desarrollar CHC es mayor. Los métodos de imagen seccional como la tomografía y resonancia magnética permiten un correcto diagnóstico al observar el patrón característico de realce arterial intenso, con lavado en fase venoportal y, en ocasiones, una cápsula de mayor densidad en secuencias tardías. Actualmente, la tomografía por emisión de positrones, que fusiona el análisis metabólico de la medicina nuclear con la imagen seccional, nos ha permitido etapificar de una manera más precisa los pacientes con CHC y dar seguimiento para la respuesta al tratamiento. El empleo de radiofármacos, como la 18-fluorodesoxiglucosa y el 1-carbono, ha demostrado que podría servir como herramienta pronóstica al aportar información indirecta sobre la diferenciación tumoral. El desarrollo futuro de nuevas tecnologías diagnósticas y el empleo de nuevos radiofármacos abre nuevas posibilidades en el diagnóstico y seguimiento por imagen de pacientes con $\mathrm{CHC}$.

Palabras clave: Carcinoma hepatocelular. Nódulo de regeneración. Nódulo displásico. PET-CT. Ácido gadoxético. 


\section{ABSTRACT}

Hepatocellular carcinoma (HCC) is currently the sixth most frequent neoplasm worldwide and the third cause of death related to cancer. The survival rate depends among other things on tumor burden and size, in addition to an opportune treatment. Actually, ultrasound is the main screening tool in patients with cirrhotic liver with risk of developing HCC. Sectional imaging such as tomography and magnetic resonance allows diagnostic with the presence of intense arterial enhancement, lavage in venoportal phase and sometimes a higher density capsule in late sequences. Currently, positron emission tomography fuses the metabolic analysis with the sectional image, allowing an accurate staging in patients with HCC and monitor the response to treatment. Radiotracers like 18-fluorodeoxyglucose and 11-carbon are useful prognostic tools and provide indirect information on tumor differentiation. The future development of diagnostic technologies and the use of new radiotracers opens up new possibilities in the diagnosis and monitoring of patients with HCC.

Key words: Hepatocellular carcinoma. Dysplastic nodule. Regenerative nodule. PET-CT. Gadoxetic acid.

\section{INTRODUCCIÓN}

El carcinoma hepatocelular (CHC) es actualmente la sexta neoplasia maligna más frecuente a nivel mundial y constituye la tercera causa de muerte relacionada con el cáncer, con una mayor incidencia en pacientes varones entre los 30 y los 50 años ${ }^{1,2}$.

A nivel mundial hay una asociación importante con la infección por el virus de la hepatitis (la hepatitis B es causante de hasta el 50\% de los casos de CHC a nivel mundial), entre otros factores de riesgo principales tenemos el hígado graso y un consumo de alcohol elevado, y en menor frecuencia hemocromatosis, obesidad, diabetes, aflatoxinas, entre otros ${ }^{3,4}$. Del 80 al 90\% de los casos asociados a infección por virus de la hepatitis B y C se reportan en pacientes con hígado cirrótico, la presencia de hígado cirrótico se asocia a un riesgo anual del 2 a $6 \%$ de desarrollar $\mathrm{CHC}^{5}$.

Existen tres patrones principales de presentación: lesión única y de gran tamaño, múltiples lesiones nodulares o una lesión difusa.

La tasa de supervivencia depende en gran parte de la carga y tamaño tumoral, así como del tratamiento oportuno, en pacientes con CHC en etapas tempranas se ha reportado una sobrevida a 5 años del 80 a 90\% de los pacientes tratados mediante trasplante hepático, y del 70\% en aquellos manejados con ablación local. La supervivencia disminuye drásticamente en pacientes que no reciben tratamiento (5\% de sobrevida a 5 años) ${ }^{6-8}$.

Por todo lo anterior, los métodos de imagen tienen un papel primordial en el diagnóstico 
oportuno de $\mathrm{CHC}$, por lo que es importante que tanto el clínico como el radiólogo conozcan los métodos diagnósticos disponibles y las guías de manejo en pacientes con factores de riesgo, con la finalidad de diagnosticar la enfermedad en etapas tempranas y, con ello, incrementar la tasa de éxito.

\section{PRESENTACIÓN CLÍNICA}

En etapas iniciales, la presentación puede ser asintomática o mostrar síntomas inespecíficos como dolor en el cuadrante superior derecho, pérdida de peso, anorexia, dolor abdominal, vómito, fiebre y fatiga. Los pacientes con daño hepático crónico pueden presentarse con ictericia, encefalopatía o anasarca. La presencia de cualquiera de estos síntomas en un paciente con antecedente de cirrosis debe levantar sospecha.

En pacientes con estadios más avanzados de daño hepático crónico, el desarrollo de hipertensión portal puede condicionar la aparición de esplenomegalia y circulación colateral (Fig. 1).

Dentro de los marcadores séricos la $\alpha$-fetoproteína (AFP) es el biomarcador más ampliamente conocido y estudiado. Consiste en una glicoproteína sintetizada por el hígado durante el desarrollo embrionario que es indetectable durante la vida adulta de un paciente sano. La elevación de AFP se asocia a un incremento en el riesgo de aparición de CHC, con una sensibilidad del 39 al 65\%, especificidad del 76 al $94 \%$ y un valor predictivo positivo del 9 al 50\%9. Se ha utilizado un valor de corte por arriba de $20 \mathrm{ng} / \mathrm{ml}$ para la detección de $\mathrm{CHC}$, aun con ello, el 20\% de los

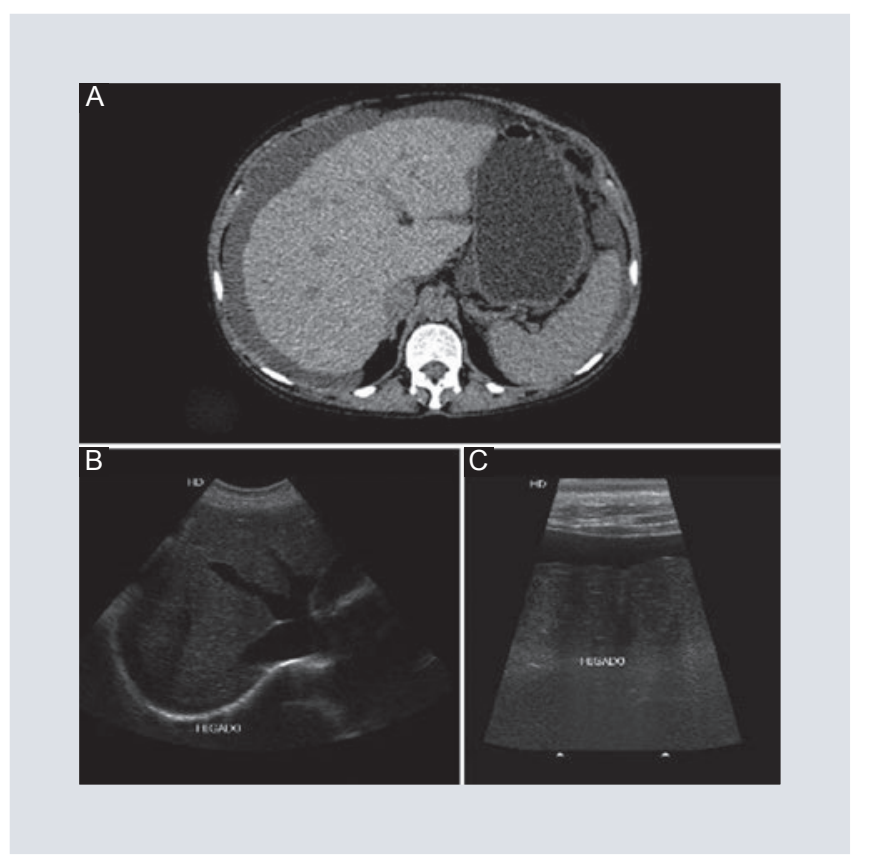

Figura 1. Paciente con hígado cirrótico. A: La tomografía axial computarizada simple muestra bordes lobulados y líquido en ambos hipocondrios; B y C: El ultrasonido presenta hígado de bordes lobulados y líquido libre perihepático.

pacientes presentan valores séricos normales. Otros estudios han sugerido valores por arriba de 100 a $200 \mathrm{ng} / \mathrm{ml}$ con el fin de incrementar la especificidad de la prueba ${ }^{10}$. Un metaanálisis publicado por Tzartzeva, et al. demostró que hay un incremento en la detección de $\mathrm{CHC}$ realizando un ultrasonido con medición de la AFP en conjunto, al comparar con pacientes en quienes se realizó solo ultrasonido como método de seguimiento ${ }^{11}$.

Otros marcadores como la Des- $\gamma$ carboxiprotrombina, Lens culinaris y la $\alpha$-Lfucosidasa se han empleado en el diagnóstico de $\mathrm{CHC}$, pero no los abordaremos en la presente revisión.

\section{HEPATOCARCINOGÉNESIS}

Los ciclos repetitivos de daño y muerte celular en pacientes con hígado cirrótico provocan 
daño en la información genética que deriva en la transformación maligna de células precancerosas.

Los nódulos de regeneración o nódulos cirróticos, por métodos de imagen presentan un flujo sanguíneo y realce similar al parénquima circundante, ya que su aporte vascular es dado principalmente por el sistema venoso portal. No se ha podido demostrar su relación con el desarrollo de CHC, aunque algunos estudios señalan que presentan un bajo riesgo de transformación maligna ${ }^{12-14}$. Por imagen son indistinguibles de los nódulos displásicos de bajo grado.

Los nódulos displásicos consisten en hepatocitos que no cumplen los criterios definitivos de malignidad, pueden ser de bajo o alto grado, dependiendo de su nivel de atipia al examen microscópico. Los nódulos displásicos de alto grado presentan características muy similares al CHC bien diferenciado por métodos de imagen. Se consideran lesiones precursoras, ya que los pacientes con nódulos de alto grado muestran cuatro veces mayor riesgo de desarrollar $\mathrm{CHC}^{13-15}$.

Se conoce como CHC temprano la lesión nodular menor de $2 \mathrm{~cm}$, con datos de atipia, que mediante microscopio presenta invasión estromal, también se cataloga como carcinoma in situ o microvascular. A diferencia de los nódulos displásicos de alto grado, pueden invadir los tractos portales y septos fibrosos, aunque no muestran un comportamiento agresivo. A mayor tamaño, el CHC se asocia a mayor grado histológico, invasión vascular y metástasis. El cambio de vasculatura portal hacia arterial provoca el realce característico en estudios contrastados. La aparición en un hígado cirrótico de una lesión nodular con realce arterial y lavado en fase venoportal es altamente sospechosa de $\mathrm{CHC}^{13,15}$.

\section{MÉTODOS DE IMAGEN}

\section{Ultrasonido}

Método de elección para el seguimiento de pacientes con cirrosis o daño hepático crónico. Según las guías de la American Association for the Study of Liver Diseases (AASLD) $y$ la European Association for the Study of the Liver (EASL) deberá realizarse un ultrasonido abdominal cada 6 meses en pacientes con factores de riesgo para $\mathrm{CHC}$, con o sin medición complementaria de $\alpha$-fetoproteína (recomendación AASLD) ${ }^{16,17}$. La EASL recomienda, ante la detección de un nódulo menor de $1 \mathrm{~cm}$ en un hígado cirrótico, realizar seguimiento cada 4 meses durante el primer año, y después cada 6 meses para valorar la estabilidad de la lesión ${ }^{16}$. La presencia de un nódulo mayor de $1 \mathrm{~cm}$ en un paciente con hígado cirrótico exige realizar estudios de extensión con tomografía axial computarizada (TAC) o resonancia magnética (RM) contrastadas ${ }^{16,17}$.

En cuanto a sus características por ultrasonido, el CHC típicamente se ha descrito como una lesión hipoecoica, aunque su ecogenicidad puede ser variable, algunos estudios han demostrado la superioridad del modo Doppler poder al comparar con el Doppler color en la detección de vascularidad intratumoral ${ }^{18}$ (Fig. 2).

La implementación del ultrasonido contrastado ha incrementado la tasa de detección de CHC respecto al ultrasonido convencional, con una precisión similar a la mostrada por 


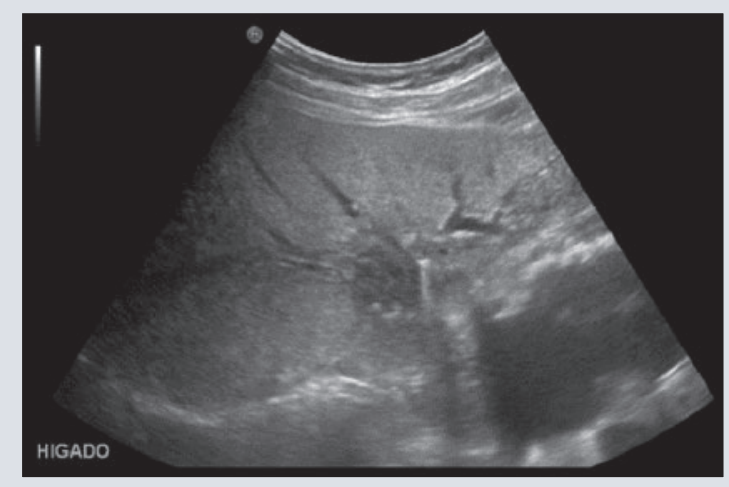

Figura 2. Lesión de predominio hipoecoica, adyacente al hilio hepático en un paciente con antecedente de hígado cirrótico. Este hallazgo obliga a la realización de un estudio dinámico contrastado para descartar sospecha de carcinoma hepatocelular.

la tomografía contrastada ${ }^{19,20}$. La última revisión de Liver Imaging Reporting and Data System (LI-RADS) v2017 incluye este método como herramienta diagnóstica de $\mathrm{CHC}^{21}$.

Como es bien conocido, la efectividad en la detección de lesiones por ultrasonido presenta una estrecha relación con la experiencia y habilidad del operador, por lo que los resultados pueden ser variables.

\section{Tomografía axial computarizada y resonancia magnética contrastadas}

En la mayoría de las guías actuales, la TAC y la RM son el método de elección para el diagnóstico de CHC. Actualmente se recomienda realizar biopsia solo en aquellos nódulos en los que no pueda determinarse benignidad o malignidad por métodos de imagen, lo que disminuye el riesgo de siembra tumoral.

En la TAC, la mayoría de los nódulos sospechosos de CHC se observan de baja densidad

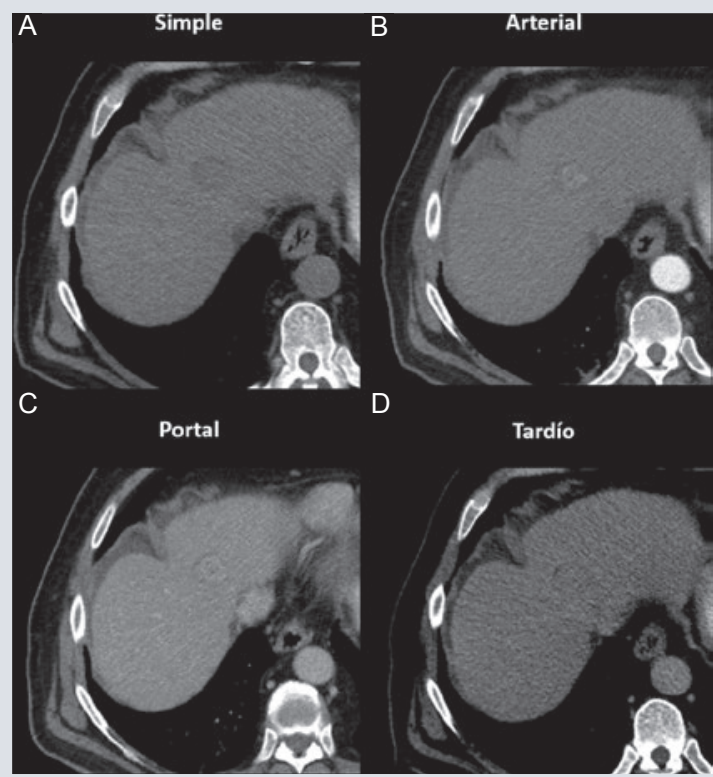

Figura 3. Tomografía axial computarizada (TAC) contrastada en paciente con antecedente de cirrosis hepática y lesión altamente sospechosa de carcinoma hepatocelular (CHC) detectada por ultrasonido. A: La TAC simple mostró una lesión hipodensa, de bordes bien definidos; B: En fase arterial presentó realce en su porción central, de mayor densidad respecto al parénquima sano; C: En la fase portal se observó isodenso al tejido adyacente; D: La fase tardía presentó lavado.

respecto al parénquima hepático normal en fase no contrastada. Tras la administración de medio de contraste, el hallazgo clásico es la presencia de un realce arterial con lavado en fase venoportal, sobre todo en tumores de gran tamaño puede observarse una cápsula de mayor densidad que se hace más evidente en fases tardías ${ }^{16}$ (Fig. 3).

La RM ha adquirido un papel importante en la detección del $\mathrm{CHC}$, con una sensibilidad del 97.4 y especificidad de $100 \%{ }^{22}$. En estudios no contrastados, el CHC clásicamente se ha descrito como una lesión hipointensa en secuencias T1 e hiperintensa en T2 respecto al parénquima hepático normal, aunque lesiones de mayor tamaño presentan aspecto más heterogéneo por la acumulación de lípidos, 


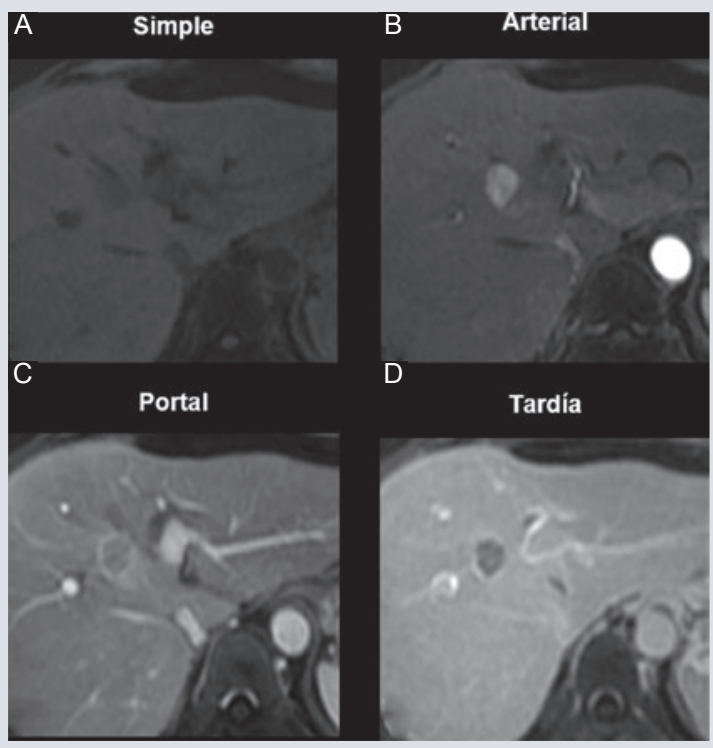

Figura 4. Resonancia magnética (RM) contrastada con gadolinio en paciente con diagnóstico de carcinoma hepatocelular. A: RM simple en secuencia T1 donde se observó una lesión nodular, hipointensa de bordes bien definidos de $1.6 \mathrm{~cm}$ de diámetro; B: En fase arterial hay intenso realce; C: Lavado característico en fase portal; D: En la fase tardía hay mayor lavado de la lesión y realce capsular.

entre otros elementos, en secuencias de difusión muestran restricción (hiperintenso) ${ }^{23}$. Tras la administración de medio de contraste extracelular pueden tener un realce similar al descrito en estudios de tomografía con realce arterial intenso a los 35 segundos y lavado rápido en fase venoportal (hipointenso respecto al parénquima hepático normal) ${ }^{16}$.

Aunque este sea el patrón característico de realce, hasta un 20\% de los CHC tienen una presentación atípica ${ }^{24}$ (Fig. 4).

Otros hallazgos por imagen que incrementan la sospecha de malignidad son el umbral de crecimiento y el tamaño de la lesión, estas características, junto con las mencionadas previamente, son consideradas como criterios de malignidad en la edición de LI-RADS v2017²5.

\section{Contrastes hepatoespecíficos en resonancia magnetica}

La mayoría de los medios de contraste empleados en la actualidad en RM están basados en gadolinio, una sustancia paramagnética capaz de modificar los tiempos de relajación T1. Por su biodistribución, los medios de contraste se subdividen en: extracelulares y hepatoespecíficos. Dentro de los medios de contraste hepatoespecíficos, actualmente está aprobado el uso de gadoxetato disódico y ácido gadoxético, ambos pueden diferenciar lesiones que contengan hepatocitos funcionales de las que no, y presentan eliminación tanto por vía biliar como urinaria ${ }^{26}$. En nuestro medio, el más empleado es el ácido gadoxético, que posee una eliminación del 50\% por la vía biliar ${ }^{27}$.

Los medios de contraste hepatoespecíficos permiten la adquisición de imágenes durante las fases «convencionales» similares a los medios de contraste extracelulares, además de una fase hepatobiliar a los $20 \mathrm{~min}$ con el ácido gadoxético o de 1 a $2 \mathrm{~h}$ con el gadoxetato disódico ${ }^{28}$. Durante esta fase hepatobiliar, las lesiones que contengan hepatocitos funcionales concentrarán medio de contraste, mientras que el CHC pobremente diferenciado se mostrará hipointenso respecto al parénquima hepático. Otras lesiones sólidas hepáticas que pueden presentarse hipointensas en fase hepatobiliar son el hemangioma, adenoma $y$ metástasis (Fig. 5).

Las principales indicaciones de uso de los medios de contraste hepatoespecíficos son la caracterización de lesiones indeterminadas mayores de $2 \mathrm{~cm}$ que no puedan caracterizarse como benignas o malignas con medios de 

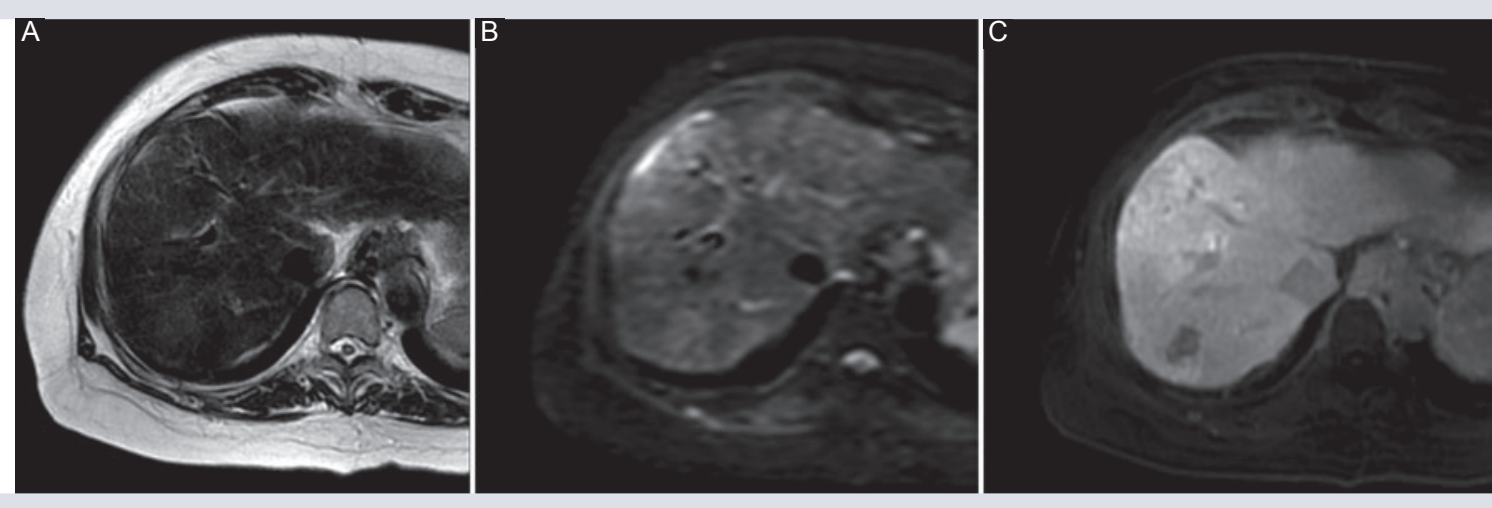

Figura 5. A: Resonancia Magnética (RM) en secuencia T2, lesión poco definida en la interlinea de los segmentos 7 y 8 con restricción molecular a la difusión; B y C: Tras la inyección de medio de contraste hepatoespecífico y a los 20 minutos (fase de equilibrio) la lesión es hipointensa al comparar con el parénquima hepático normal lo que comprueba la ausencia de hepatocitos funcionales.

contraste convencionales y en el abordaje inicial de lesiones menores de $2 \mathrm{~cm}^{26}$.

\section{Tomografía por emisión de positrones}

La tomografía por emisión de positrones (PET) fusiona el análisis metabólico de la medicina nuclear con un método de imagen como es la TAC. Mediante el uso de radiotrazadores, el PET-CT busca medir la actividad de un fármaco radiomarcado y administrado vía endovenosa en el paciente.

El trazador más ampliamente utilizado es la fluorodesoxiglucosa (FDG) marcada con flúor $18\left({ }^{18} \mathrm{~F}\right)$, este radiotrazador permite valorar la actividad metabólica de la glucosa con una vida media de 110 min. En el paciente con $\mathrm{CHC}$ el ${ }^{18} \mathrm{~F}$-FDG presenta una baja sensibilidad (40 a 50\%), que se ve afectada por la elevada actividad metabólica del parénquima hepático sano ${ }^{29}$. Los tumores menos diferenciados y con una mayor tasa de replicación celular tienden a mostrar mayor actividad metabólica, por lo que captan más ávidamente el radiofármaco ${ }^{30}$. Es importante conocer que el ${ }^{18} \mathrm{~F}-\mathrm{FDG}$ no es un marcador exclusivo de actividad tumoral y se ve incrementado en procesos infecciosos o inflamatorios.

El otro fármaco empleado en nuestro medio en pacientes con $\mathrm{CHC}$ es el acetato marcado con carbono $11\left({ }^{11} \mathrm{C}\right)$, este fármaco ingresa al ciclo de Krebs como sustrato de la $\beta$-oxidación en la síntesis de ácidos grasos y colesterol, ambos están incrementados en pacientes con carcinoma hepatocelular bien diferenciado, por lo que es útil en estadios más tempranos de la enfermedad, su vida media es de $20 \mathrm{~min}^{31}$. La sensibilidad reportada en la literatura del ${ }^{11} \mathrm{C}$-acetato es de entre el 80 a $90 \%{ }^{29}$.

La sensibilidad del radiotrazador es dependiente de la diferenciación celular, mientras que el ${ }^{11} \mathrm{C}$-acetato detecta de mejor manera tumores de bajo grado (bien diferenciados), el ${ }^{18}$ F-FDG es superior en tumores de alto grado (pobremente diferenciados), por lo cual se ha sugerido el uso de ambos radiotrazadores de forma complementaria ${ }^{30}$. Estudios como el realizado por Park, et al. han concluido que la realización de un protocolo 


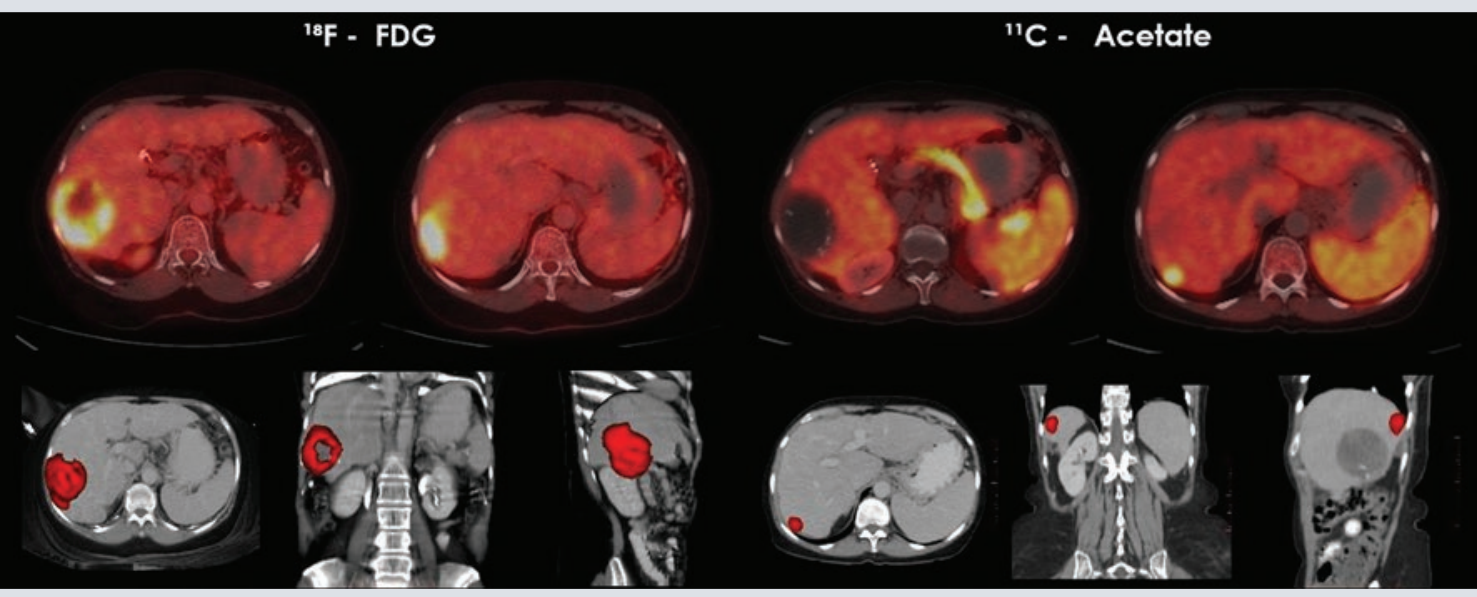

Figura 6. Paciente de 69 años con lesión hepática que muestra captación periférica de fluorodesoxiglucosa marcada con flúor 18 $\left({ }^{18} \mathrm{~F}-\mathrm{FDG}\right)$ en el segmento VII. El estudio con acetato marcado con carbono 11 ("1'C-acetato) realizado una semana después no mostró captación de esta lesión, pero se identificó una segunda lesión en el segmento VI no se había visto previamente.

dual $\left({ }^{18} \mathrm{~F}-\mathrm{FDG}\right.$ y ${ }^{11} \mathrm{C}$-acetato) en pacientes con sospecha de CHC, incrementa la sensibilidad para la detección de lesiones primarias, aunque la detección de lesiones menores de $1 \mathrm{~cm}$ aún es baja ${ }^{31}$. Debido a su relación con tumores poco diferenciados, la mayor captación de ${ }^{18} \mathrm{~F}$-FDG se ha considerado un factor de peor pronóstico y sobrevida para el paciente $^{31}$ (Fig. 6).

Los sitios preferidos de metástasis a distancia en el CHC son el pulmón, hueso, glándula suprarrenal, peritoneo y cerebro. De los dos radiofármacos, el ${ }^{18} \mathrm{~F}$-FDG presenta una mayor sensibilidad en la detección de metástasis a distancia, mientras el ${ }^{11} \mathrm{C}$-acetato tiene mejor especificidad, muy útil para la detección de ganglios sospechosos. En un estudio realizado por Ho, et al., el empleo de ambos radiofármacos en pacientes con metástasis de CHC reportó una sensibilidad y especificidad global del 98 y $86 \%$, respectivamente ${ }^{32}$.

Una desventaja del protocolo con dos radiofármacos es el costo elevado, por lo que no es una técnica muy ampliamente utilizada en países en vías de desarrollo. Como alternativa en el abordaje de $\mathrm{CHC}$ menor de $3 \mathrm{~cm}$ algunos estudios han empleado un protocolo dual solo utilizando ${ }^{11} \mathrm{C}$-acetato, este cosiste en una fase de adquisición temprana del abdomen superior, inmediata tras la administración del radiotrazador, y la fase convencional que se realiza a los 18 min posteriores a la inyección. Pacientes con carcinoma hepatocelular bien diferenciado presentan señal elevada en la fase temprana la cual incrementa en etapas tardías. Lesiones benignas como la hiperplasia nodular focal y hemangiomas muestran realce positivo en fase temprana y una disminución de la actividad en la segunda fase $e^{33}$.

\section{DIAGNÓSTICO OPORTUNO}

La importancia de un diagnóstico oportuno recae en que un tratamiento temprano se asocia a una mayor tasa de curación, ya sea mediante tratamiento con resección quirúrgica, 
trasplante o ablación percutánea. La supervivencia a 5 años es del $75 \%$ de los pacientes que son tratados de manera oportuna ${ }^{34}$.

Los métodos de imagen tienen un papel importante en la detección de todas las lesiones tumorales y en su medición, factores importantes en la elección terapéutica de los pacientes.

Tanto los criterios de Milán como la clasificación de Barcelona toman en cuenta el tamaño tumoral, el número de lesiones, la invasión vascular y la metástasis a distancia para clasificar a los pacientes con CHC y determinar el tratamiento más efectivo ${ }^{34,35}$. El mayor tamaño tumoral se ha asociado a un incremento en la falla al tratamiento o recurrencia tumoral postrasplante.

\section{CONCLUSIONES}

El papel del ultrasonido sigue siendo fundamental en el screening de pacientes con factores de riesgo conocidos, aunque su sensibilidad está relacionada a factores del paciente, experiencia del operador y tamaño del tumor.

Los métodos de imagen seccional han demostrado una gran precisión en la detección de tumores con patrones de realce característicos, la identificación de una lesión hepática que presente realce en fase arterial con lavado tardío y cápsula, en el contexto de un paciente con hígado cirrótico hace confiable el diagnóstico de CHC sin la necesidad de una biopsia confirmatoria. El empleo de contrastes hepatoespecíficos por RM en lesiones de comportamiento dudoso ha mejorado la precisión diagnóstica.
El PET-CT mediante el empleo dual de ${ }^{18}$ F-FDG $y{ }^{11} \mathrm{C}$-acetato se ha posicionado entre las principales herramientas diagnósticas, ya que permite la valoración tanto funcional como por imagen de las lesiones hepáticas, con una elevada sensibilidad en el diagnóstico de enfermedad metastásica y una buena especificidad en la detección de ganglios sospechosos.

El empleo actual del PET-CT y el desarrollo de nuevos radiofármacos abre un futuro esperanzador en la detección en etapas tempranas de pacientes con $\mathrm{CHC}$, así como la detección de la totalidad de las lesiones que permita brindar un mejor abordaje terapéutico que impactaría en una mejor calidad de vida.

\section{CONFLICTO DE INTERESES}

Los autores declaran que no existe conflicto de interés.

\section{FINANCIAMIENTO}

Los autores declaran que no se requirió financiamiento especial para la realización del presente trabajo.

\section{RESPONSABILIDADES ÉTICAS}

Protección de personas y animales. Los autores declaran que para esta investigación no se han realizado experimentos en seres humanos ni en animales.

Confidencialidad de los datos. Los autores declaran que han seguido los protocolos de 


\author{
su centro de trabajo sobre la publicación de \\ datos de pacientes.
}

\section{Derecho a la privacidad y consentimiento informado. Los autores han obtenido el con- sentimiento informado de los pacientes y/o sujetos referidos en el artículo. Este docu- mento obra en poder del autor de correspon- dencia.}

\section{BIBLIOGRAFÍA}

1. Shariff MI, Cox IJ, Gomaa AI, Khan SA, Gedroyc W, Taylor-Robinson SD. Hepatocellular carcinoma: current trends in worldwide epidemiology, risk factors, diagnosis and therapeutics. Expert Rev Gastroenterol Hepatol. 2009;3:353-67.

2. Ghouri YA, Mian I, Rowe JH. Review of hepatocellular carcinoma: Epidemiology, etiology, and carcinogenesis. J. Carcinog. 2017;16:1.

3. El-Serag HB. Hepatocellular Carcinoma. N. Engl. J. Med. 2011;365:1118-27.

4. Parkin DM. The global health burden of infection-associated cancers in the year 2002. Int J Cancer. 2006;118:3030-44.

5. Zhang DY, Friedman SL. Fibrosis-dependent mechanisms of hepatocarcinogenesis. Hepatology. 2012;56:769-75.

6. Livraghi T, Meloni F, Di Stasi M, Rolle E, Solbiati L, Tinelli C, Rossi S. Sustained complete response and complications rates after radiofrequency ablation of very early hepatocellular carcinoma in cirrhosis: Is resection still the treatment of choice? Hepatology. 2007;47:82-9.

7. Roayaie S, Blume IN, Thung SN, Guido M, Fiel MI, Hiotis S, et al. A system of classifying microvascular invasion to predict outcome after resection in patients with hepatocellular carcinoma. Gastroenterology. 2009; 137:850-5.

8. Roayaie S, Obeidat K, Sposito C, Mariani L, Bhoori S, Pellegrinelli A, et al. Resection of hepatocellular cancer $\leq 2 \mathrm{~cm}$ : results from two Western centers. Hepatology. 2013;57:1426-35.

9. Daniele B, Bencivenga A, Megna AS,Tinessa, V. $\alpha$-fetoprotein and ultrasonography screening for hepatocellular carcinoma. Gastroenterology. 2004;127:S108-12.

10. Ahn DG, Kim HJ, Kang H, Lee HW, Bae SH, Lee JH, et al. Feasibility of $\alpha$-fetoprotein as a diagnostic tool for hepatocellular carcinoma in Korea. Korean J Intern Med. 2016;31:46-53.

11. Tzartzeva K, Obi J, Rich NE, Parikh ND, Marrero JA, Yopp A, et al. Surveillance Imaging and Alpha Fetoprotein for Early Detection of Hepatocellular Carcinoma in Patients With Cirrhosis: A Meta-analysis. Gastroenterology. 2018;154:1706-18.e1.

12. Sato T, Kondo F, Ebara M, Sugiura N, Okabe S, Sunaga M, et al. Natural history of large regenerative nodules and dysplastic nodules in liver cirrhosis: 28-year follow-up study. Hepatol Int. 2015;9:330-6.

13. Cassinotto C, Aubé C, Dohan A. Diagnosis of hepatocellular carcinoma: An update on international guidelines. Diagn. Interv. Imaging. 2017;98:379-91.

14. Röcken C, Carl-McGrath S. Pathology and Pathogenesis of Hepatocellular Carcinoma. Dig Dis. 2001;19:269-78.

15. Roskams T, Kojiro M. Pathology of Early Hepatocellular Carcinoma: Conventional and Molecular Diagnosis. Semin Liver Dis. 2010;30:017-25.
16. European Association For The Study Of The Liver \& European Organisation For Research And Treatment Of Cancer. EASL-EORTC Clinical Practice Guidelines: Management of hepatocellular carcinoma. J. Hepatol. 2012:56:908-43

17. Heimbach JK, Kulik LM, Finn RS, Sirlin CB, Abecassis MM, Roberts LR, et al. AASLD Guidelines for the Treatment of Hepatocellular Carcinoma. Hepatology. 2018;67(1):358-80.

18. Ignee A, Weiper D, Schuessler G, Teuber G, Faust D, Dietrich CF. Sonographic Characterisation of Hepatocellular Carcinoma at Time of Diagnosis. Z Gastroenterol. 2005;43:28.94.

19. Chen RC, Chen WT, Liao LY, Cheng NY, Wang CK, Tu HY, et al. Intravenous contrast-enhanced doppler sonography and intra-arterial carbon dioxide- enhanced sonography in the assessment of hepatocellular carcinoma vascularity before and after treatment. Acta Radiol. 2002;43:411-4.

20. Fiore F, Vallone P, Ricchi P, Tambaro R, Daniele B, Sandomenico F, et al. Levovist-enhanced Doppler Sonography Versus Spiral Computed Tomography to Evaluate Response to Percutaneous Ethanol Injection in Hepatocellular Carcinoma. J Clin Gastroenterol. 2000;31:164-68.

21. LI-RADS ${ }^{\circledR}$ v2017 CEUS Core CEUS Diagnostic Table.

22. Matos AP, Velloni F, Ramalho M, AlObaidy M, Rajapaksha A, Semelka RC. Focal liver lesions: Practical magnetic resonance imaging approach. World J Hepatol. 2015;7:1987-2008.

23. Khosa F, Khan AN, Eisenberg RL. Hypervascular liver lesions on MRI. Am J Roentgenol. 2011;197(2):W204-20.

24. D'Ippolito G, Fernando Appezzato L, Caivano RAC, de Abreu L Junior, Borri ML, Galvão Filho MM. Apresentações incomuns do hepatocarcinoma: ensaio iconográfico. Radiol Bras. 2006;39:137-43.

25. Elsayes KM, Hooker JC, Agrons MM, Kielar AZ, Tang A, Fowler KJ, et al. 2017 Version of LI-RADS for CT and MR Imaging: An Update. Radiographics. 2017;37:1994-2017.

26. Cossio-Torrico PE, Ramírez-Carmonab CR, Stoopen-Romettic M, Perochena-González A, Sosa-Lozanod LA, E. Kimura-Hayamac E, et al. Resonancia magnética con ácido gadoxético — contraste hepatoespecífico- para la evaluación de lesiones focales. Rev Gastroenterol México. 2015;80:267-75.

27. Van Beers BE, Pastor CM, Hussain HK. Primovist, Eovist: What to expect? J. Hepatol. 2012;57:421-9.

28. Motosugi U, Ichikawa T, Tominaga L, Sou H, Sano K, Ichikawa S, et al. Delay before the hepatocyte phase of Gd-EOB-DTPA-enhanced MR imaging: Is it possible to shorten the examination time? Eur Radiol. 2009;19:2623-9.

29. Ho CL, Yu SCH, Yeung DWC. 11C-acetate PET imaging in hepatocellular carcinoma and other liver masses. J Nucl Med. 2003;44:213-21.

30. Larsson P, Arvidsson D, Björnstedt M, Isaksson B, Jersenius U, Motarjemi H. Adding 11C-acetate to 18F-FDG at PET Examination Has an Incremental Value in the Diagnosis of Hepatocellular Carcinoma. Mol Imaging Radionucl Ther. 2012;21:6-12.

31. Park JW, Kim JH, Kim SK, Kang KW, Park KW, Choi JI, et al. A Prospective Evaluation of 18F-FDG and 11C-Acetate PET/CT for Detection of Primary and Metastatic Hepatocellular Carcinoma. J Nucl Med. 2008;49:1912-23.

32. Ho CL, Chen S, Yeung, DW, Cheng TK. Dual-Tracer PET/CT Imaging in Evaluation of Metastatic Hepatocellular Carcinoma. J Nucl Med. 2007;48:902-9.

33. Huo L, Dang Y, Lim F. Application of dual phase imaging of 11C-acetate positron emission tomography on differential diagnosis of small hepatic lesions. Mol Imaging Biol. 2015;17.

34. Mazzaferro V, Regalia E, Doci R, Andreola S, Pulvirenti A, Bozzetti F, et al. Liver Transplantation for the Treatment of Small Hepatocellular Carcinomas in Patients with Cirrhosis. N Engl J Med. 1996;334:693-700.

35. Jianyong L, Lunan Y, Wentao W, Yong Z, Bo L, Tianfu W, et al. Barcelona Clinic Liver Cancer Stage B Hepatocellular Carcinoma. Medicine (Baltimore). 2014;93:e180. 